\title{
Microfluidic magnetic separator using an array of soft magnetic elements
}

\author{
Smistrup, Kristian; Lund-Olesen, Torsten; Hansen, Mikkel Fougt; Tang, Peter Torben
}

Published in:

Journal of Applied Physics

Link to article, DOI:

$10.1063 / 1.2159418$

Publication date:

2006

Document Version

Publisher's PDF, also known as Version of record

Link back to DTU Orbit

Citation (APA):

Smistrup, K., Lund-Olesen, T., Hansen, M. F., \& Tang, P. T. (2006). Microfluidic magnetic separator using an array of soft magnetic elements. Journal of Applied Physics, 99(8), 08P102. https://doi.org/10.1063/1.2159418

\section{General rights}

Copyright and moral rights for the publications made accessible in the public portal are retained by the authors and/or other copyright owners and it is a condition of accessing publications that users recognise and abide by the legal requirements associated with these rights.

- Users may download and print one copy of any publication from the public portal for the purpose of private study or research.

- You may not further distribute the material or use it for any profit-making activity or commercial gain

- You may freely distribute the URL identifying the publication in the public portal 


\title{
Microfluidic magnetic separator using an array of soft magnetic elements
}

\author{
Kristian Smistrup, ${ }^{\text {a) }}$ Torsten Lund-Olesen, and Mikkel F. Hansen \\ MIC-Department of Micro and Nanotechnology, Technical University of Denmark, \\ Building 345 East, DK-2800 Kongens Lyngby, Denmark \\ Peter T. Tang \\ Department of Manufacturing Engineering and Management, Technical University of Denmark, \\ Building 424, DK-2800 Kongens Lyngby, Denmark
}

(Presented on 2 November 2005; published online 17 April 2006)

\begin{abstract}
We present the design, fabrication, characterization, and demonstration of a new passive magnetic bead separator. The device operates in an effective state when magnetized by an external magnetic field of only $50 \mathrm{mT}$, which is available from a tabletop electromagnet. We demonstrate the complete capture of $1.0 \mu \mathrm{m}$ fluorescent magnetic beads from a $7.5 \mu \mathrm{L}$ sample volume traveling at an average linear fluid velocity of $5 \mathrm{~mm} / \mathrm{s}$. (C) 2006 American Institute of Physics. [DOI: 10.1063/1.2159418]
\end{abstract}

\section{INTRODUCTION}

Recently several magnetic bead separators in microfluidic formats have been reported in the literature. Most use active on-chip microelectromagnets, ${ }^{1-6}$ while others use passive designs where on-chip soft magnetic structures are magnetized by external magnetic fields. ${ }^{7-9}$ While designs using on-chip electromagnets generally produce small magnetic fields in the microfluidic channels, the passive designs generally provide larger fields and gradients in the channel.

We present a microsystem with a passive magnetic separator featuring an array of electroplated long soft magnetic elements facing the microfluidic channel. Capture of fluorescent magnetic beads is demonstrated using fluorescence microscopy.

\section{MICROSYSTEM FABRICATION}

Figure 1 shows a photograph of the entire chip. The microfluidic channel measures $1 \times \mathrm{h} \times \mathrm{w}=13 \quad 500 \times 80$ $\times 200 \mu \mathrm{m}^{3}(\approx 0.2 \mu \mathrm{L})$, and each of the permalloy magnetic elements measures $1 \times \mathrm{h} \times \mathrm{w}=4400 \times 50 \times 150 \mu \mathrm{m}^{3}$. The elements are placed with a periodicity of $350 \mu \mathrm{m}$ and a spacing to the channel of $20 \mu \mathrm{m}$. The long thin shape of the magnetic elements is chosen to minimize demagnetization effects and thus maximize fields and gradients.

Figure 2 summarizes the fabrication procedure. As $\mathrm{Wu}$ et al. ${ }^{10}$ we use Deep Reactive Ion Etched (DRIE) structures as electroplating molds, but we have developed the method substantially. In (a) and (b) of Fig. 2, a $9.5 \mu$ m thick layer of AZ4562 photoresist (Hoechst, NJ, USA) is patterned using UV lithography on a single polished $\mathrm{Si}$ (100) wafer. The photoresist pattern is used as an etch mask for a DRIE in (c) to create trenches for the electroplating mold as well as the microfluidic channels. In (d) the wafer is RCA cleaned, a $1 \mu \mathrm{m}$ thick $\mathrm{SiO}_{2}$ is grown on the wafer, and finally a seed layer of $5 \mathrm{~nm} \mathrm{Ti}$ and $200 \mathrm{~nm} \mathrm{Au}$ is E-beam evaporated onto the wafer.

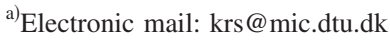

The trenches designated for electroplated magnetic elements are all connected to the outer rim of the wafer by a network of conducting paths. The poor step coverage obtained by the metal E-beam evaporation ensures that little or no contact exists between the metal in the conducting network and the metal on top of the wafer. Short etches of $\mathrm{Au}$ are performed in Entreat 100 (Engelhard, NJ, USA) until no contact persists between the top and the bottom. A $2.5 \mu \mathrm{m}$ thick protective $\mathrm{Cu}$ layer is electroplated in the bottom of the conducting network using the commercially available $\mathrm{Cu}$ proStar LP-1 electrolyte (Enthone, CT, USA). Subsequently, the unprotected $\mathrm{Au}$ and $\mathrm{Ti}$ is etched away in Entreat 100 and $5 \%$ hydrofluoric acid, respectively. Finally, the $\mathrm{Cu}$ layer is removed in nitric acid.

At this point the wafer features the microfluidic channels and the conducting network. Permalloy $\left(\mathrm{Ni}_{80} \mathrm{Fe}_{20}\right)$ is electroplated into the network, including the trenches for magnetic elements using a pulse-reversal plating electrolyte as described in Ref. 11. The resulting structure is shown in (e). In (f) a pyrex lid is bonded anodically to the top of the wafer, sealing the microfluidic channel. When the wafer is diced into chips, the conducting paths are cut away, leaving only the soft magnetic elements on the chip. Access to the microfluidic channel is gained by drilling holes in the pyrex wafer.

\section{EXPERIMENTAL DETAIL}

Magnetic hysteresis measurements on an entire microsystem and the magnetic beads were performed in a LakeShore 7407 vibrating sample magnetometer (VSM).

The bead capture experiments were carried out with FITC fluorescence labeled $1.0 \mu \mathrm{m}$ magnetic beads (FCM 1052-2, Spherotec, IL, USA). A solution of 7 $\times 10^{7}$ beads $/ \mathrm{mL} \mathrm{H}_{2} \mathrm{O}$ was injected into the microfluidic channel using a syringe pump at a flow rate of $5 \mu \mathrm{L} / \mathrm{min}$.

With the bead solution flowing in the channel pictures were taken starting at time $t=0$. At $t=10 \mathrm{~s}$ a tabletop electromagnet supplying a homogeneous field perpendicular to the fluid channel was switched on at its maximum field of $50 \mathrm{mT}$. At $t=100 \mathrm{~s}$ the field was switched off. 


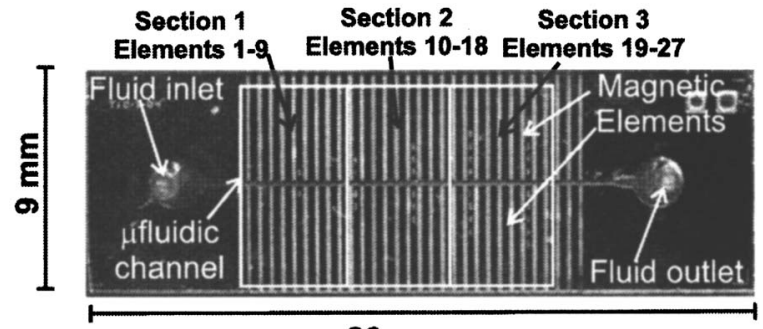

$26 \mathrm{~mm}$

FIG. 1. Picture of the magnetic separation chip showing the position of the microfluidic channel and the passive soft magnetic elements. The external homogenous magnetic field is applied vertically in the plane of the paper.

The bead capture in the microfluidic channel was quantified at different times using the FITC fluorescence images taken every second with an exposure time of $0.9 \mathrm{~s}$ on a Leica MZ FL III microscope equipped with a Sony DFW-X710 CCD camera. Images were taken for each of the channel sections defined in Fig. 1 and the fluorescence signal from each section was quantified using MATHEMATICA as follows: First, the color micrographs were converted to gray scale by summing the RGB values pixel by pixel. Then the intensity values of all pixels having an intensity larger than a threshold value of 45 were summed to give the fluorescence signal of the specific section. This value was chosen by trial and error and is necessary to filter out the background noise from dark pixels.

\section{RESULTS AND DISCUSSION}

Figure 3 shows the magnetic hysteresis curve of the microsystem. The saturation flux density of Permalloy $\left(\mathrm{Ni}_{80} \mathrm{Fe}_{20}\right)$ is approximately $1 \mathrm{~T}$. An applied field of $\mu_{0} \mathrm{H}$ $\approx 20 \mathrm{mT}$ is sufficient to saturate the magnetic elements. The coercive field is $\mu_{0} \mathrm{H}_{c} \approx 0.5 \mathrm{mT}$, and the remanence relative to saturation is $\approx 25 \%$, ensuring that the beads are released when the external field is switched off.

Figure 4 shows the magnetic hysteresis curve of the beads. It is seen that the beads are saturated in a field of $250 \mathrm{mT}$ and that the applied field of $50 \mathrm{mT}$ brings the beads to $50 \%$ of their saturation value. Also, these beads show a
A)

B)

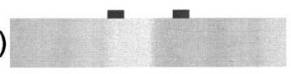

C)

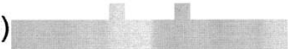

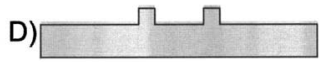

E)

F)

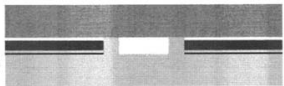

FIG. 2. Fabrication scheme (cross-section view). (a) shows the starting double polished Si wafer. In (b) a photoresist mask is applied using UV lithography. In (c) trenches for both the microfluidic channel and magnetic elements are etched. RCA-clean, oxide growth $(1 \mu \mathrm{m}$, wet), and seed layer deposition $(5 / 200 \mathrm{~nm} \mathrm{Ti} / \mathrm{Au}$, E-beam evaporation) with intentionally low step coverage is performed in (d). The areas designated for magnetic elements are connected through conducting paths and isolated from the rest of the wafer. In (e) a thin layer of $\mathrm{Cu}$ is electroplated in these places, and the seed layer is removed on the rest of the wafer using the $\mathrm{Cu}$ layer as etch mask. After $\mathrm{Cu}$-removal permalloy are electroplated into the conducting paths and magnetic elements. After seed layer and oxide removal a pyrex lid is bonded anodically to the wafer in (f).

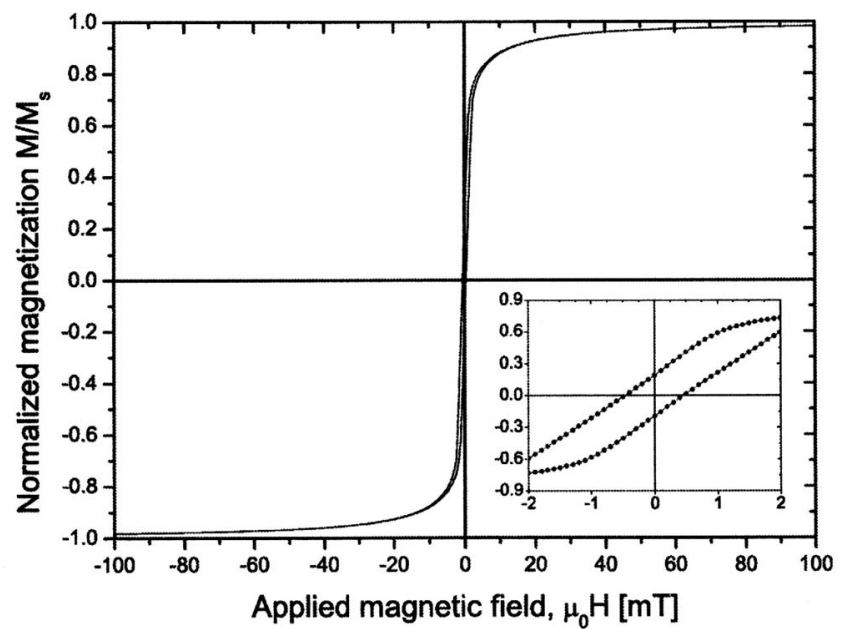

FIG. 3. Normalized magnetic hysteresis curve of the magnetic separation chip. The inset shows the low-field region.

considerable amount of hysteresis, which is unusual for magnetic beads that are normally considered to be ensembles of superparamagnetic particles.

The magnetic force, $\boldsymbol{F}$, on a magnetic bead is given by

$$
\begin{aligned}
\boldsymbol{F} & =\mu_{0} \int_{\text {Bead }}\left\{\boldsymbol{M}\left[\boldsymbol{H}_{0}(\boldsymbol{x})\right] \cdot \boldsymbol{\nabla}\right\} \boldsymbol{H}_{0}(\boldsymbol{x}) \mathrm{d} v \\
& \approx \mu_{0} V_{b}\left\{\boldsymbol{M}\left[\boldsymbol{H}_{0}\left(\boldsymbol{x}_{b}\right)\right] \cdot \boldsymbol{\nabla}\right\} \boldsymbol{H}_{0}\left(\boldsymbol{x}_{b}\right),
\end{aligned}
$$

where $\mu_{0}$ is the magnetic permeability of vacuum, $\boldsymbol{M}$ is the magnetization of the magnetic bead, and $\boldsymbol{H}_{0}$ is the magnetic field in the absence of a magnetic bead. $V_{b}$ and $\boldsymbol{x}_{b}$ are the volume and position of the magnetic bead, respectively. ${ }^{12}$

Equation (1) shows that the magnetic force is proportional to the magnetic field gradient, which originates from the magnetic elements, since the applied field is homogeneous. The force is also proportional to the magnetization of the magnetic bead (assuming the approximation that the angle between the magnetization and the applied field does not change substantially when the field is increased.)

Earlier, we have seen that the soft magnetic elements are saturated by an external magnetic field of $50 \mathrm{mT}$, and that

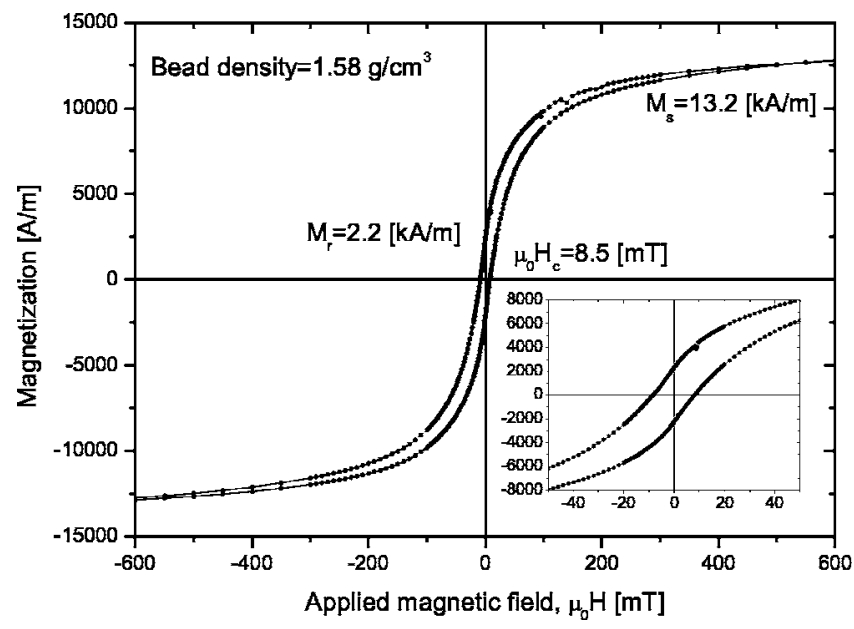

FIG. 4. Magnetic hysteresis curve for the $1.0 \mu \mathrm{m}$ fluorescent magnetic beads (Spherotec FCM-1052-2). The inset shows the low-field region. 


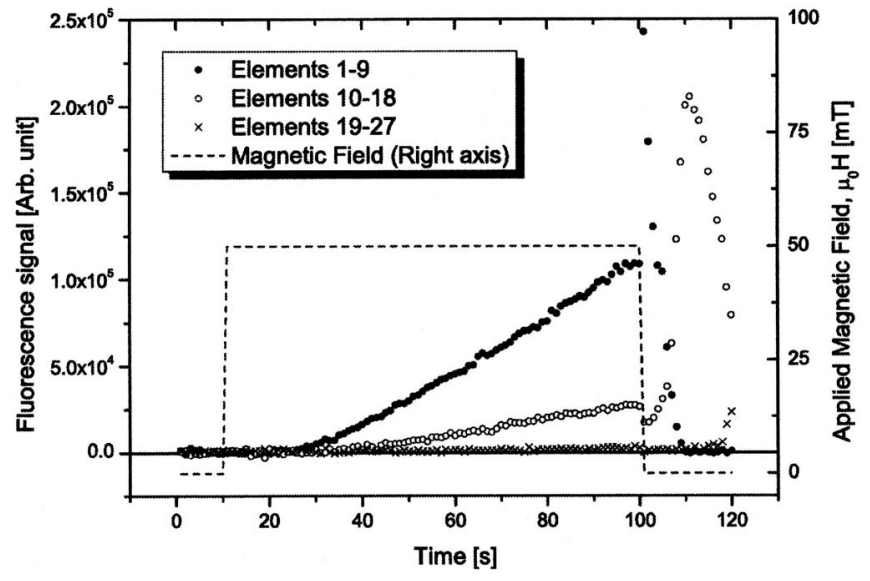

FIG. 5. The applied magnetic field and the measured fluorescent signal for the three sections of the microfluidic channel as a function of time.

the same field will bring a magnetic bead within $50 \%$ of its saturation magnetization. This means that increasing the applied field beyond $50 \mathrm{mT}$ will not affect the gradient, but it will bring the magnetization of the beads closer to saturation. Together Figs. 3 and 4 show that using a larger applied field could ultimately give a less than twofold increase in the magnetic forces and bead capture efficiency in the microfluidic channel.

Figure 5 shows the quantified fluorescence signals for the three channel sections as a function of time. Most beads are captured on elements 1-9 (large signal rise), some are captured on elements 10-18 (smaller signal rise), and none are captured at elements 19-27 (zero signal rise). The fact that no significant fluorescence signal is observed from elements 19-27 while the magnet is on indicates that the amount of beads that are not captured in the first two sections is negligible and hence that we have complete bead capture. When the magnetic field is turned off at $t=100 \mathrm{~s}$, large peaks appear in the fluorescence signals from the three sections after which the signals return to the baseline, indicating that all beads have been released. This is seen most clearly for elements 1-9.

Compared to the design of Deng et al. with $\mathrm{Ni}$ post inside the fluidic channel, ${ }^{7}$ our design has the advantage that the magnetic material is located outside the channel and that both an effective bead capture and release is achieved. Compared to Ichikawa et al., ${ }^{8}$ who use sputtered thin films, our magnetic elements are thicker and hence influence a larger region of space with a potentially better bead capture. Compared to the design of Rida and Gijs, ${ }^{9}$ our system has more capture points, allowing for higher capture efficiency. As it is fabricated using Si MEMS technology, there is better control of dimensions allowing for smaller magnetic element to channel separation and potentially higher magnetic forces, and the process has a greater potential for integration with other functionalities and batch production.

We have demonstrated a new passive magnetic bead separator and demonstrated that it effectively captures magnetic beads from a bead solution traveling at an average linear velocity of $5 \mathrm{~mm} / \mathrm{s}(5 \mu \mathrm{L} / \mathrm{min})$ in the field supplied from a small tabletop electromagnet. At this flow rate, however, only two-thirds of the channel length participate in the capture, so the system is expected to be effective up to even higher flow rates. Furthermore, we have demonstrated that the beads can be released after capture. This makes the system reusable and suited for use in a portable lab-on-a-chip system.

\section{ACKNOWLEDGMENTS}

Danish Technological Institute and the Copenhagen Graduate School for Nanoscience and Nanotechnology (C:O:N:T) are acknowledged for financially supporting Kristian Smistrup's Ph.D. studies.

${ }^{1}$ K. Smistrup, O. Hansen, H. Bruus, and M. F. Hansen, J. Magn. Magn. Mater. 293, 597 (2005).

${ }^{2}$ C. H. Ahn, M. G. Allen, W. Trimmer, Y.-N. Jun, and S. Erramilli, J. Microelectromech. Syst. 5, 151 (1996).

${ }^{3}$ J.-W. Choi, K. W. Oh, A. Han, C. A. Wijayawardhana, C. Lannes, S. Bhansali, K. T. Schlueter, W. R. Heineman, H. B. Halsall, J. H. Nevin, et al., Biomed. Microdevices 3, 191 (2001).

${ }^{4}$ R. Rong, J.-W. Choi, and C. H. Ahn, in Proceedings IEEE Sixteenth Annual International Conference on Micro Electro Mechanical Systems, MEMS-03 Kyoto, Japan, January 19-23 (IEEE, New York, 2003), p. 530533.

${ }^{5}$ M. Tondra, M. Granger, R. Fuerst, M. Porter, C. Nordman, J. Taylor, and S. Akou, IEEE Trans. Magn. 37, 2621 (2001).

${ }^{6}$ Q. Ramadana, V. Samperb, D. Poenara, and C. Yub, J. Magn. Magn. Mater. 281, 150 (2004)

${ }^{7}$ T. Deng, M. Prentiss, and G. M. Whitesides, Appl. Phys. Lett. 80, 461 (2002).

${ }^{8}$ N. Ichikawa, Y. Katsuyama, Y. Nagasaki, and T. Ichiki, in Proceedings of the 8th International Conference on Miniaturized Systems in Chemistry and Life Sciences ( $\mu$ TAS), 2004, Vol. 2, p. 384.

${ }^{9}$ A. Rida and M. A. M. Gijs, Anal. Chem. 76, 6239 (2004).

${ }^{10}$ J. Wu, V. Quinn, and G. H. Bernstein, J. Micromech. Microeng. 14, 576 (2004).

${ }^{11}$ P. T. Tang, in Proceedings of SUR/FIN 2001, Nashville, June 25-28, 2001.

${ }^{12}$ A. Engel and R. Friedrichs, Am. J. Phys. 70, 428 (2002). 